\title{
In-orbit magnetometer bias and scale factor calibration
}

\author{
Chingiz Hajiyev ${ }^{\star}$ \\ Faculty of Aeronautics and Astronautics, Istanbul Technical University, Istanbul, Turkey
}

Received: 26 February 2016 / Accepted: 6 March 2016

\begin{abstract}
Magnetometers are widely used for LEO small satellites attitude determination and control system. In order to estimate satellite dynamics and control attitude accurately, scale factor and bias of magnetometer must be estimated. In this study a linear Kalman filter (LKF) based algorithm for the estimation of magnetometer biases and scale factors is proposed. Proposed algorithms are simulated through attitude dynamics of a small satellite.
\end{abstract}

Keywords: Magnetometer, calibration, Kalman filtering, satellite, attitude determination

\section{Introduction}

Magnetometers are widely used for small satellites attitude determination and control system. These sensors provide the direction of magnetic field and its magnitude, therefore they are useful for attitude determination and control. Moreover, their low mass and low power consumtion make them attractive for small satellites. For these reasons, most LEO small satellites have magnetometers as part of their basic sensor package. In order to estimate the components of the geomagnetic field vector for attitude determination and attitude control accurately, effects of measurement bias and scale factor should be canceled.

In last decade, popularity of small satellites with low cost and weight has increased significantly. That brought about a search for lighter but more accurate sensors. Under these circumstances, three-axis magnetometer (TAM) has become so attractive because of its advantages such as providing a continuously available two-axis attitude measurements; relative low cost and almost insignificant power demand.

Operating magnetometers as primary sensor in small satellite missions is a common method for achieving attitude information. However, these sensors are not error free because of the biases, scaling errors and misalignments (nonorthagonality). These terms inhibit the filter efficiency and so attitude data accuracy and even they may bring about the filter divergence in long terms. The attitude accuracy requirements demand compensation for the magnetometer errors such as misalignments, scale factors and biases [1]. Estimating magnetometer biases and scale factors as well as the attitude of the satellite is a proposed technique to solve such problems and increase on-board accuracy.

\footnotetext{
${ }^{\star}$ Correspondence: cengizprof@gmail.com
}

One method to calibrate magnetometer measurements is to follow up an attitude free scheme. In usual cases, magnetometers may be the only operating sensors at the orbit injection phase where attitude data is not available since the spacecraft is spinning rapidly. Besides, magnetometer measurements are not bias free at that stage because of the large magnetic disturbances on the spacecraft such as charging during the launch and the electrical currents within the spacecraft [2]. Hence, magnetometers must be calibrated without the knowledge of attitude in such cases.

In literature there are several methods for estimating the magnetometer bias in case of lack of attitude knowledge. Bias vector can be basically solved by minimizing the weighted sum of the squares of residuals which are the differences in the squares of the magnitudes of the measured and modelled magnetic fields [1]. However, this procedure results with a cost function which is quartic in the magnetometer bias vector and in order to avoid the prone to error minimization process of the quartic function of the magnetometer bias, a number of alternate methods have been developed. In their papers, Shuster and Alonso propose a relatively new algorithm called twostep, which combines the convergence in a single step of a heuristic algorithm with the correct treatment of the statistics of the measurement [2-4]. In reference [5] they present a modified twostep algorithm that estimates all three components of the magnetometer bias vector in case where the centered portion of the negative-log-likelihood function provides incomplete observability of the magnetometer bias vector. Besides, in reference [6] it is shown that twostep algorithm can be also used to estimate scale factors and nonorthogonality corrections as well as magnetometer biases by an attitude-independent procedure. As another implication example of twostep algorithm, Kim and Bang integrates it with the genetic algorithm and uses the genetic algorithm for providing the initial estimates of the magnetometer bias estimation [7]. Twostep algorithm like methods can 
be used for guessing an initial estimate for Kalman filter type stochastic attitude determination methods. Those a priori estimates for the estimator may be then corrected on an expanded state vector including attitude parameters and biases.

It is possible to also meet with other attitude-free magnetometer bias estimation methodologies in literature. In reference [8], it is proved that a full magnetometer calibration can be performed on-orbit during typical spacecraft mission-mode operations by the use of realtime algorithms based on both the extended Kalman filter (EKF) and unscented Kalman filter (UKF). Even as, in reference [9] EKF is utilized for real-time three axis magnetometer calibration as well as the low Earth orbit determination. Although these studies can estimate the magnetometer characteristics such as biases, scale factors and nonorthogonality corrections, they all disregard the attitude dynamics of the satellite and if this information is possible at any instant, their accuracies can be exceeded. Hence they may be considered as a part of spacecraft modes where attitude data is absent.

In reference [10] both the magnetometer biases and scale factors are estimated by recursive least squares method. This algorithm is a recursive implementation of the least squares minimization technique. The vector of unknown parameters is formed of magnetometer biases and scale factors. Method is not attitude free since the attitude knowledge is necessary to form the attitude matrix. However as a drawback, used attitude parameters are not estimated by the method itself and the spacecraft dynamics are not taken into consideration in this method. On the other hand, a few studies like $[11,12]$, which consider magnetometer bias and scale factor estimates as a part of attitude estimation process, also do exist. They handle magnetometer calibration process together with the estimation of attitude parameters. In reference [11] EKF is used as a part of the estimation scheme. Via an UKF based estimation algorithm, which has advantages over EKF such as absence of Jacobian calculations etc., its accuracy may be surmounted. Ma and Jiang [12] solve magnetometer calibration and attitude estimation problem via two UKFs working synchronously. As a disadvantage, this approach requires a high computational effort because of two distinct UKFs and it may be not suitable for pico and nano satellites where the processing capacity of the attitude computer is limited.

The magnetometer zero drift is estimated in reference [13] via a linear Kalman filter (LKF) during the orbital movement of picosatellite. In this work an algorithm for calibrating the magnetometer biases using the parameters characterizing the dynamics of the rotational motion of a picosatellite is proposed. However, the magnetometer scale factor calibration problrm is not taken into consideration in this study.

In order to obtain accurate geomagnetic field measurements, the sensor should be calibrated precisely. In this study a LKF based calibration algorithm is proposed for in-orbit magnetometer bias and scale factor estimation. This algorithm is suitable for pico and nano satellites which has three magnetometers as measurement sensors and the processing capacity of the attitude computer is limited.

The structure of this paper is as follows. In Section 2, the magnetometer measurement model is described. Design of LKF for estimation of magnetometer biases and scale factors is presented in Section 3. In Section 4, some simulations are carried out for the LKF based magnetometer calibration algorithms. Section 5 gives a brief summary of the obtained results and conclusions.

\section{The magnetometer measurement model}

As the satellite navigates along its orbit, magnetic field vector differs in a relevant way with the orbital parameters. If those parameters are known, then, magnetic field tensor vector that affects satellite can be shown as a function of time analytically [14]. Note that, these terms are obtained in the orbit reference frame.

$$
\begin{aligned}
B_{o x}(t)= & \frac{M_{e}}{r_{0}^{3}}\left\{\cos \left(\omega_{0} t\right)[\cos (\varepsilon) \sin (i)\right. \\
& \left.-\sin (\varepsilon) \cos (i) \cos \left(\omega_{e} t\right)\right] \\
& \left.-\sin \left(\omega_{0} t\right) \sin (\varepsilon) \sin \left(\omega_{e} t\right)\right\} \\
B_{o y}(t)= & -\frac{M_{e}}{r_{0}^{3}}\left[\cos (\varepsilon) \cos (i)+\sin (\varepsilon) \sin (i) \cos \left(\omega_{e} t\right)\right] \\
B_{o z}(t)= & \frac{2 M_{e}}{r_{0}^{3}}\left\{\sin \left(\omega_{0} t\right)[\cos (\varepsilon) \sin (i)\right. \\
& \left.-\sin (\varepsilon) \cos (i) \cos \left(\omega_{e} t\right)\right] \\
& \left.-2 \sin \left(\omega_{0} t\right) \sin (\varepsilon) \sin \left(\omega_{e} t\right)\right\}
\end{aligned}
$$

here:

- $M_{e}=7.943 \times 10^{15} \mathrm{~Wb} . \mathrm{m}$; the magnetic dipole moment of the Earth,

$-i=97^{\circ}$; the orbit inclination,

$-\omega_{e}=7.29 \times 10^{-5} \mathrm{rad} / \mathrm{s}$; the spin rate of the Earth,

$-\varepsilon=11.7^{\circ}$; the magnetic dipole tilt,

$-\omega_{0}$; the angular velocity of the orbit with respect to the inertial frame, found $\operatorname{as} \omega_{0}=\left(\mu / r_{0}^{3}\right)^{1 / 2}$,

$-\mu=3.98601 \times 10^{14} \mathrm{~m}^{3} / \mathrm{s}^{2}$; the Earth Gravitational constant,

$-r_{0}=6,928,140 \mathrm{~m}$; the distance between the centre of mass of the satellite and the Earth.

Three onboard magnetometers of satellite measures the components of the magnetic field vector in the body frame. Therefore for the measurement model, which characterizes the measurements in the body frame, gained magnetic field terms must be transformed by the use of direction cosine matrix, $A$. Overall measurement model may be given as:

$$
\left[\begin{array}{c}
B_{m x}(\varphi, \theta, \psi, t) \\
B_{m y}(\varphi, \theta, \psi, t) \\
B_{m z}(\varphi, \theta, \psi, t)
\end{array}\right]=\left(S A\left[\begin{array}{l}
B_{o x}(t) \\
B_{o y}(t) \\
B_{o z}(t)
\end{array}\right]+b_{m}+v(t)\right)
$$


where, $B_{o x}(t), B_{o y}(t)$ and $B_{o z}(t)$ represent the Earth magnetic field vector components in the orbit frame as a function of time, and $B_{m x}(\varphi, \theta, \psi, t), B_{m y}(\varphi, \theta, \psi, t)$ and $B_{m z}(\varphi, \theta, \psi, t)$ show the measured Earth magnetic field vector components in body frame as a function of time and varying Euler angles. Furthermore, $S$ is the scale factor matrix as:

$$
S=\left[\begin{array}{lll}
S_{x} & 0 & 0 \\
0 & S_{y} & 0 \\
0 & 0 & S_{z}
\end{array}\right]=\operatorname{diag}\left(S_{x}, S_{y}, S_{z}\right)
$$

$b_{m}$ is the magnetometer bias vector as $b_{m}=\left[b_{m_{x}} b_{m_{y}} b_{m_{z}}\right]^{T}$ and $v(t)$ is the zero mean Gaussian white noise with the characteristic of

$$
E\left[v_{k} v_{j}^{T}\right]=I_{3 x 3} \sigma_{m}^{2} \delta_{k j}=R_{v} \delta_{k j} .
$$

$I_{3 x 3}$ is the identity matrix with the dimension of $3 \times 3, \sigma_{m}$ is the standard deviation of each magnetometer error, $R_{v}$ is the covariance matrix of the measurement noise and $\delta_{k j}$ is the Kronecker symbol.

In order to obtain high accurate magnetometer measurements, the sensor should be calibrated before mission on the ground or in-orbit. The ground calibration requires a huge calibration facility and system, magnetic shield chamber, more time and cost. Therefore, the magnetometers are calibrated on the ground coarsely, because it is difficult to calibrate attitude sensors precisely on the ground. Furthermore, launch shock vibration and post-launch disturbances can cause error of magnetometer measurement after the ground calibration.

In generally, the magnetometers are calibrated coarsely on the ground, and these bias and scale factor errors are estimated from real measurements more precisely in orbit [15]. An optimum linear Kalman filter algorithm for in-orbit magnetometer calibration of satellites is proposed below.

\section{Design of the optimum linear kalman filter for magnetometer biase and scale factor estimation}

The estimation technique for the magnetometer bias and scale factor using LKF is proposed in this section.

According to a linear observation model (4), the magnetometer measurement model can be expressed in the discrete-time form as follows:

$$
B_{m}(k)=S(k) A(k) B_{o}(k)+b(k)+v(k) .
$$

For a random walk process, the continuous-time state equations of the magnetometer bias and scale factor are given by:

$$
\begin{aligned}
\dot{b} & =u_{b} \\
\dot{S} & =u_{S}
\end{aligned}
$$

where $u_{b}$ and $u_{S}$ are the white noises with zero mean.
The state equations (8) and (9) can be written in the discrete-time form as:

$$
\begin{gathered}
b(k)=b(k-1)+T_{s} u_{b}(k-1) \\
S(k)=S(k-1)+T_{s} u_{S}(k-1)
\end{gathered}
$$

where $T_{s}$ is the sampling time, $b(k)=\left[b_{x}(k) b_{y}(k) b_{z}(k)\right]^{T}$ and $S(k)=\left[S_{x}(k) S_{y}(k) S_{z}(k)\right]^{T}$.

Let us form the expanded state vector as:

$$
\Sigma(k)=\left[b_{x}(k) b_{y}(k) b_{z}(k) S_{x}(k) S_{y}(k) S_{z}(k)\right]^{T} .
$$

Then the following equation can be written

$$
\Sigma(k)=\Sigma(k-1)+T_{s} U(k-1)
$$

where

$$
U(k)=\left[u_{b_{x}}(k) u_{b_{y}}(k) u_{b z}(k) u_{S x}(k) u_{S y}(k) u_{S z}(k)\right]^{T} .
$$

We can rewrite the equations (10) and (11) in state-space form, yielding

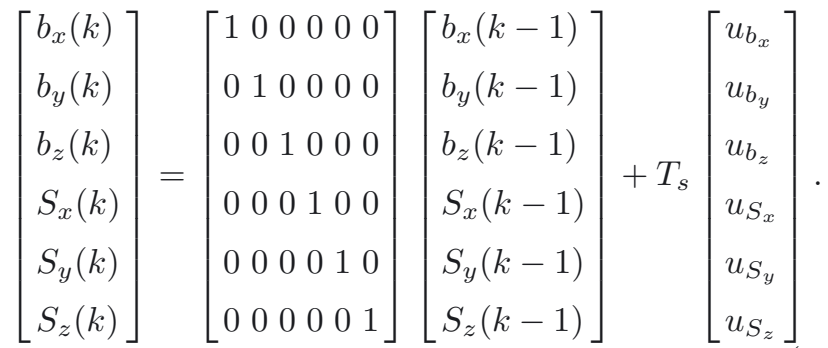

This means that the systems dynamics matrix is the $(6 \times 6)$ unit matrix.

Let's move linear state model (13) into following form:

$$
\begin{aligned}
\Sigma(k) & =\Sigma(k-1)+T_{s} U(k-1) \\
B_{m}(k) & =S(k) A(k) B_{o}(k)+b(k)+v(k) .
\end{aligned}
$$

The measurement matrix in this case is

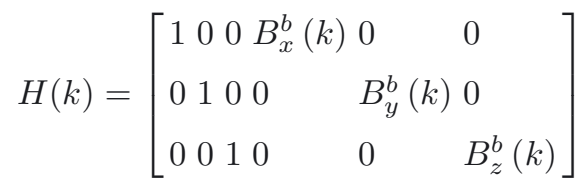

where

$$
\begin{aligned}
& B_{x}^{b}(k)=a_{11}(k) B_{o x}(k)+a_{12}(k) B_{o y}(k)+a_{13}(k) B_{o z}(k) \\
& B_{y}^{b}(k)=a_{21}(k) B_{o x}(k)+a_{22}(k) B_{o y}(k)+a_{23}(k) B_{o z}(k) \\
& B_{z}^{b}(k)=a_{31}(k) B_{o x}(k)+a_{32}(k) B_{o y}(k)+a_{33}(k) B_{o z}(k)
\end{aligned}
$$

are the components of the Earth magnetic field vector in body frame, $a_{i j},(i, j=\overline{1,3})$ are the elements of the direction cosine matrix, $A$.

Applying the Optimum Kalman filter equations [16] to model (14) and (15) we obtain the following Kalman filter 
for estimation of magnetometer bias and scale factor

$$
\begin{aligned}
\hat{\Sigma}(k)= & \hat{\Sigma}(k-1)+K(k) \\
& \times\left[B_{m}(k)-\hat{b}(k-1)-\hat{S}(k-1) A(k) B_{o}(k)\right] \\
K(k)= & P(k / k-1) H^{T}(k) \\
& \times\left[H(k) P(k / k-1) H^{T}(k)+R_{v}(k)\right]^{-1} \\
P(k / k)= & {[I-K(k) H(k)] P(k / k-1) } \\
P(k / k-1)= & P(k-1)+T_{s}^{2} Q_{U}
\end{aligned}
$$

where $\hat{\Sigma}(k)$ is the estimation value, $K(k)$ is the Kalman gain, $P(k / k)$ is the covariance matrix of the estimation error, $P(k / k-1)$ is the covariance matrix of the extrapolation error, $I$ is the identity matrix.

The innovation sequence of an optimal Kalman filter has precisely defined characteristics, which can be compared with the output of an actually implemented Kalman filter. The innovation process contains all information to assess the optimality of filter operations [17].

The innovation sequence is defined as the difference between the actual system output and the predicted output based on the predicted state. This sequence has the property below.

If the system operates normally then innovation sequence

$$
\Delta(k)=B_{m}(k)-\hat{b}(k-1)-\hat{S}(k-1) A(k) B_{o}(k)
$$

in Kalman filter which is adjusted according to the model of system dynamic will be white Gaussian noise with zeromean and covariance matrix [18];

$$
P_{\Delta}(k)=H(k) P(k / k-1) H^{T}(k)+R_{v}(k) .
$$

It is more appropriate to use normalized innovation sequence to supervise the Kalman filter operation:

$$
\tilde{\Delta}(k)=\left[H(k) P(k / k-1) H^{T}(k)+R_{v}(k)\right]^{-1 / 2} \Delta(k) .
$$

Because in this case;

$$
E\left[\tilde{\Delta}(k) \tilde{\Delta}^{T}(j)\right]=P_{\tilde{\Delta}}=I \delta(k j) .
$$

Since the normalized innovations have the distribution $N(0,1)$, they will be in the confidence interval $[-3,+3]$ with probability 0.9986 in case of optimal operation of Kalman filter.

\section{Simulations}

Simulations are realized with a sampling time of $T_{s}=$ $0.1 \mathrm{~s}$. As an experimental platform a cubesat model is used. Nonetheless the orbit of the satellite is a circular orbit with an altitude of $r=550 \mathrm{~km}$. Other orbit parameters are same as it is presented in the section for the Magnetometer Model (Sect. 2).
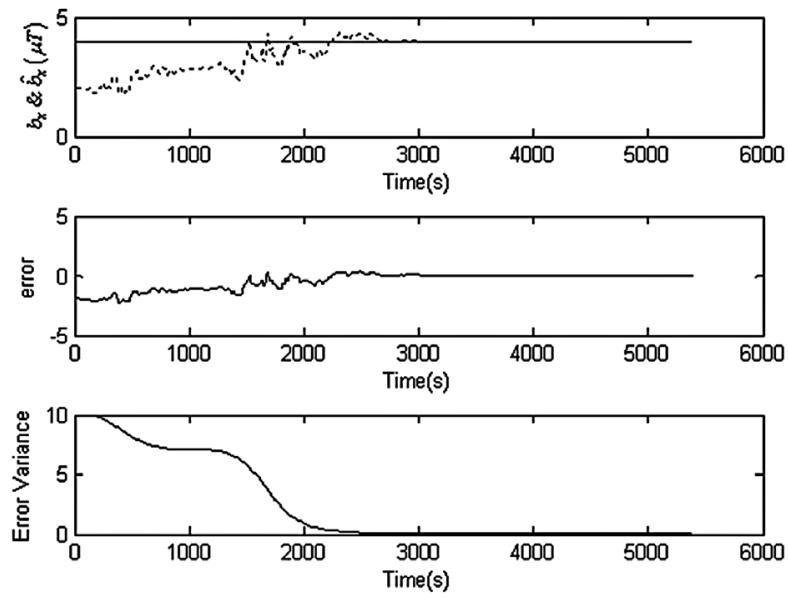

Fig. 1. Actual ( $b_{x}:$ solid line) and estimated $\left(\hat{b}_{x}\right.$ : dotted line) bias values, difference between them and error variance.
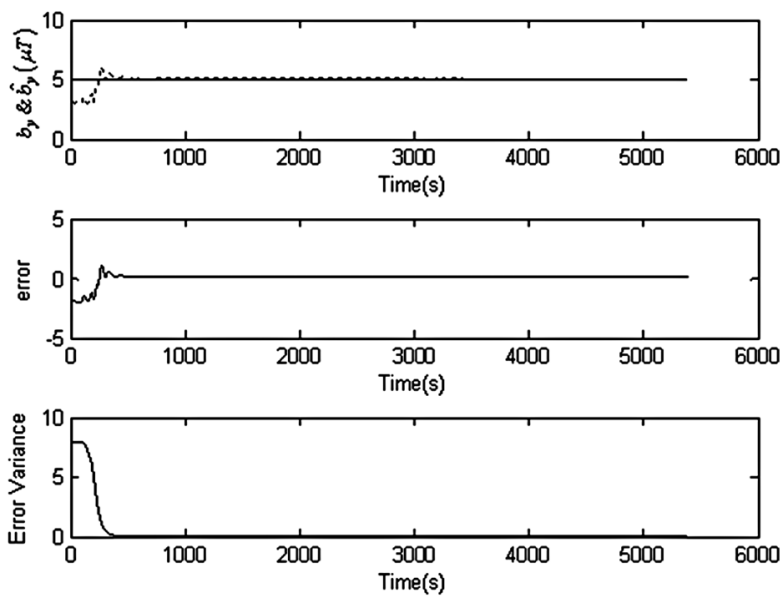

Fig. 2. Actual ( $b_{y}$ : solid line) and estimated ( $\hat{b}_{y}$ : dotted line) bias values, difference between them and error variance.

For the magnetometer measurements, sensor noise is characterized by zero mean Gaussian white noise with a standard deviation of $\sigma_{m}=0.83 \mu T$.

The magnetometer biases and scale factors estimation results are shown in Figures 1-6. In the proposed algorithm, state vector is formed of 6 states; 3 magnetometer biases and 3 scale factors. Bias and scale factor estimation results are given in Figures 1-3 and 4-6, respectively. The first part of figures gives LKF magnetometer bias (or scale factor) estimation results and the actual values in a comparing way. Second part of the figures shows the error of the estimation process based on the actual estimation values of the satellite. The last part indicates the variance of the estimation. As it is apparent, bias terms and scale factors are estimated accurately.

Behaviours of the normalized innovations of LKF are shown in Figure 7. As seen from presented graphs the normalized innovations of all measurement channels lay between the bounds of the confidence interval $[-3,+3]$.

Comparison of the calibrated and uncalibrated TAM results is shown in Figures 8-10. In these figures the true 

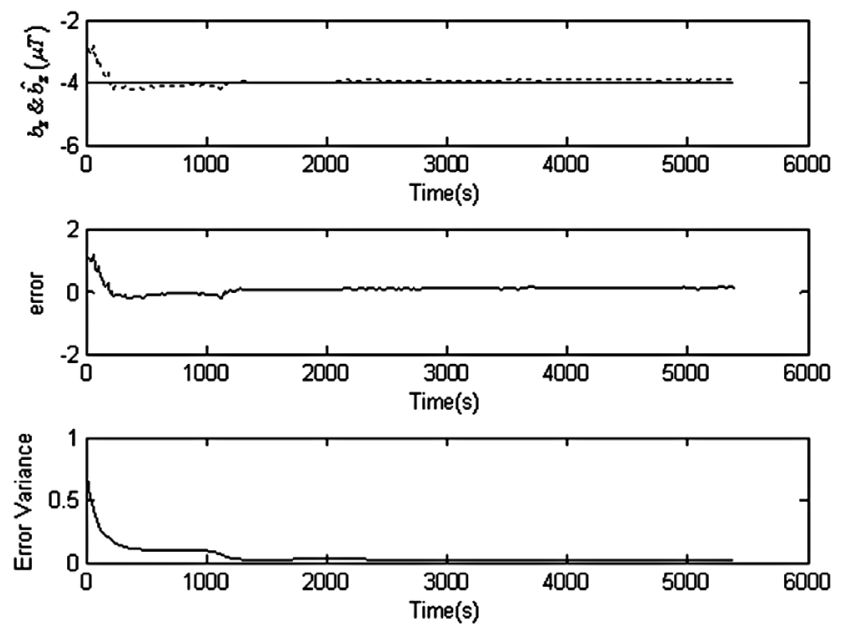

Fig. 3. Actual ( $b_{z}$ : solid line) and estimated $\left(\hat{b}_{z}\right.$ : dotted line) bias values, difference between them and error variance.
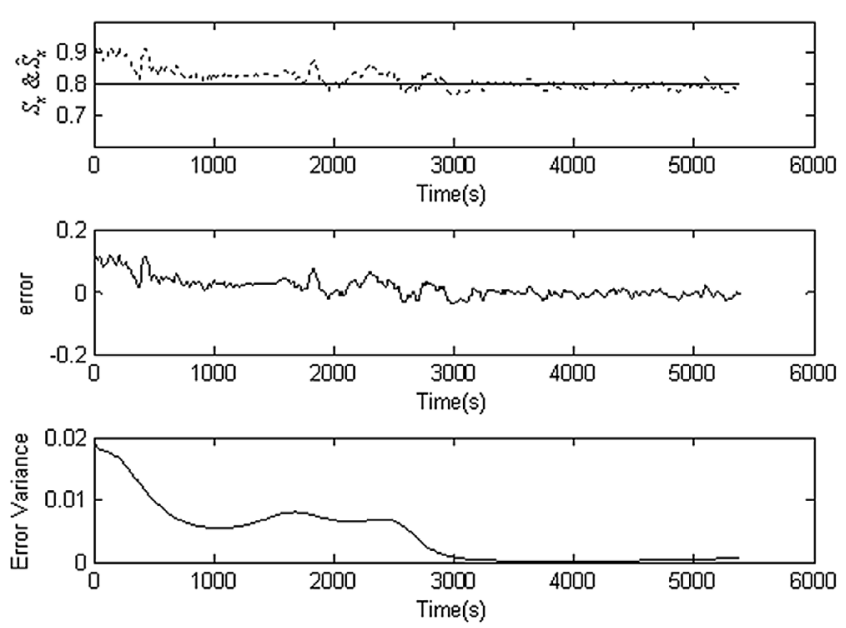

Fig. 4. Actual ( $S_{x}$ : solid line) and estimated $\left(\hat{S}_{x}\right.$ : dotted line) scale factor values, difference between them and error variance.

values of the geomagnetic field magnitudes in body frame and the calibrated and uncalibrated magnetometers measurement results are presented. As seen from figures, the calibrated magnetometer results are very close to the true values of geomagnetic field and considerably better than the uncalibrated magnetometer results. The uncalibrated results are significantly biased and scaled,but calibrated results are free from these components.

Absolute and relative estimation errors of magnetometer biases and scale factors when the proposed LKF is used are presented in Tables 1 and 2 respectively. In Table 1 the absolute estimation errors of magnetometer biases and scale factors are presented. The results in Table 2 correspond to the relative estimation errors of magnetometer biases and scale factors.

As seen from presented in Tables 1 and 2 results, the bias and scale factor estimation errors are very small after $750 \mathrm{sec}$ of estimation procedure.
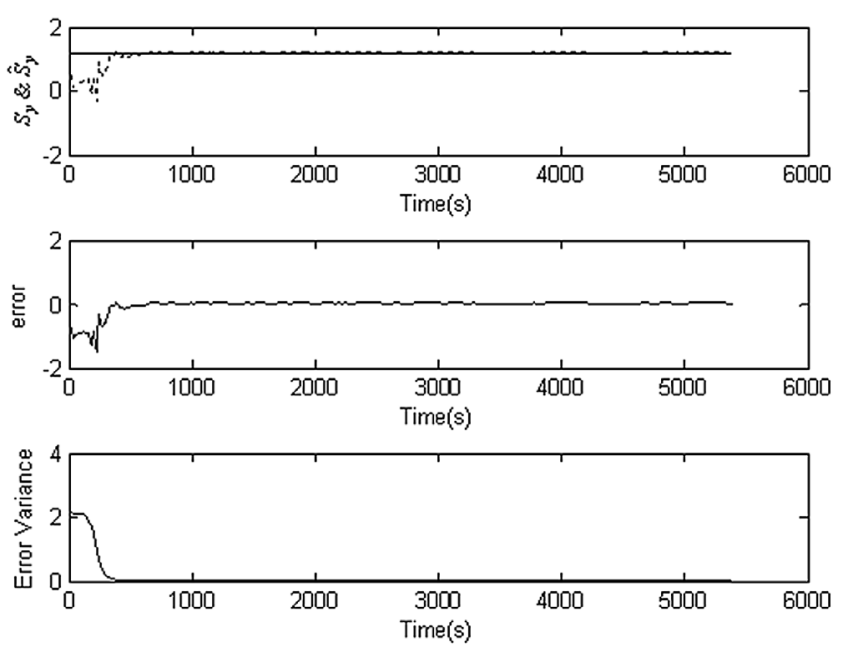

Fig. 5. Actual ( $S_{y}$ : solid line) and estimated $\left(\hat{S}_{y}\right.$ : dotted line) scale factor values, difference between them and error variance.
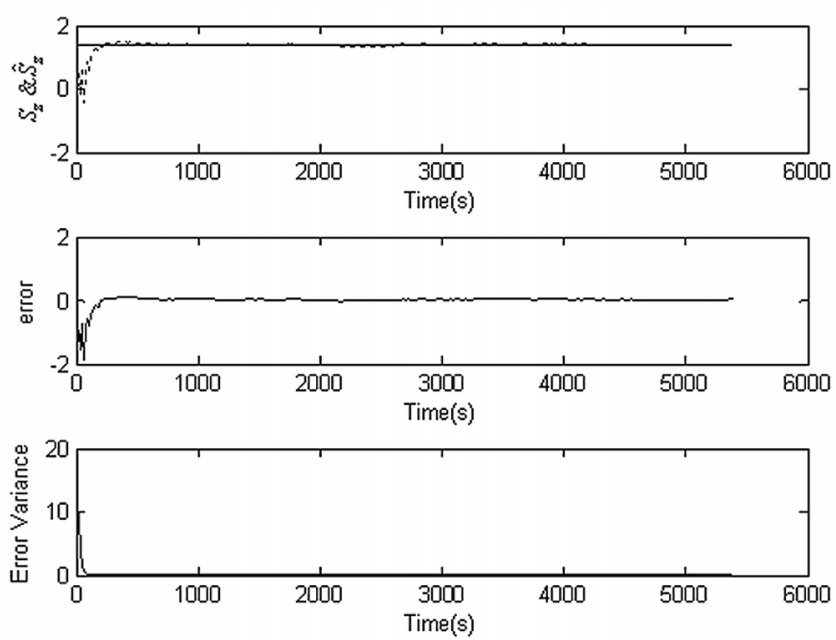

Fig. 6. Actual $\left(S_{z}\right.$ : solid line) and estimated $\left(\hat{S}_{z}\right.$ : dotted line) scale factor values, difference between them and error variance.
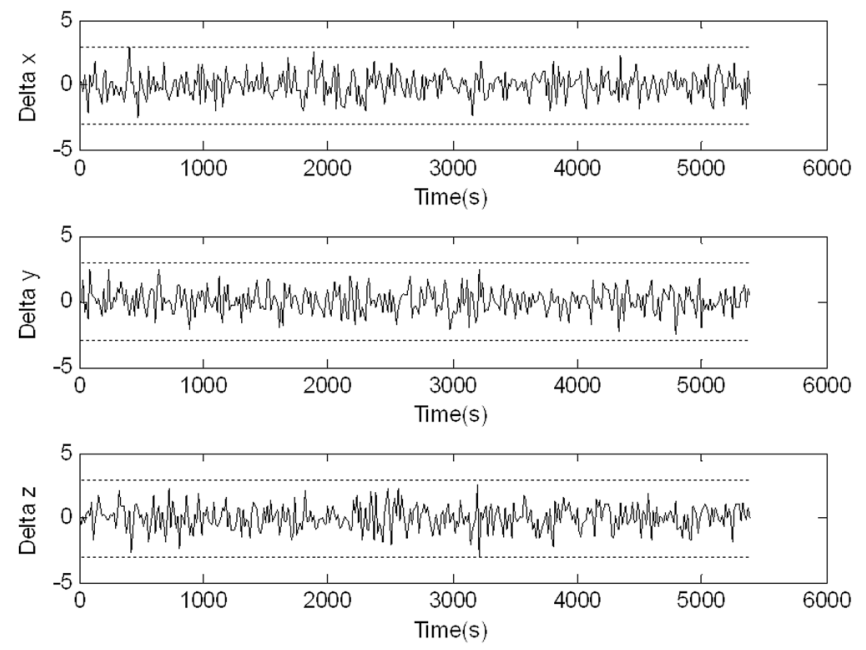

Fig. 7. Behaviors of the normalized innovations. 
Table 1. Absolute estimation errors of magnetometer biases and scale factors

\begin{tabular}{ccccccc}
\hline \multirow{2}{*}{ Time (s) } & $\begin{array}{c}\text { Abs. Error } \\
\Delta b_{x}(\mu T)\end{array}$ & $\begin{array}{c}\text { Abs. Error } \\
\Delta b_{y}(\mu T)\end{array}$ & $\begin{array}{c}\text { Abs. Error } \\
\Delta b_{z}(\mu T)\end{array}$ & $\begin{array}{c}\text { Abs. Error } \\
\Delta S_{x}\end{array}$ & $\begin{array}{c}\text { Abs. Error } \\
\Delta S_{y}\end{array}$ & $\begin{array}{c}\text { Abs. Error } \\
\Delta S_{z}\end{array}$ \\
\hline 150 & -1.9864 & -1.5691 & 0.3526 & 0.1123 & -0.9187 & -0.2604 \\
750 & -1.1442 & -0.0781 & -0.0197 & 0.0195 & -0.0106 & 0.0107 \\
1500 & -0.6148 & -0.0844 & 0.0035 & 0.0290 & -0.0099 & 0.0165 \\
2250 & 0.0099 & -0.0836 & 0.0119 & 0.0269 & 0.0150 & 0.0296 \\
3000 & 0.0038 & -0.0914 & 0.0273 & -0.0361 & 0.0101 & -0.0222 \\
3750 & -0.0105 & -0.0494 & -0.0234 & -0.0206 & -0.0046 & -0.0210 \\
4500 & -0.0118 & -0.0613 & -0.0571 & -0.0047 & -0.0113 & 0.0245 \\
5250 & -0.0048 & -0.0631 & -0.0786 & -0.0108 & 0.0254 & -0.0180 \\
5400 & 0.0020 & -0.0570 & -0.0648 & -0.0023 & -0.0151 & 0.0369 \\
\hline
\end{tabular}

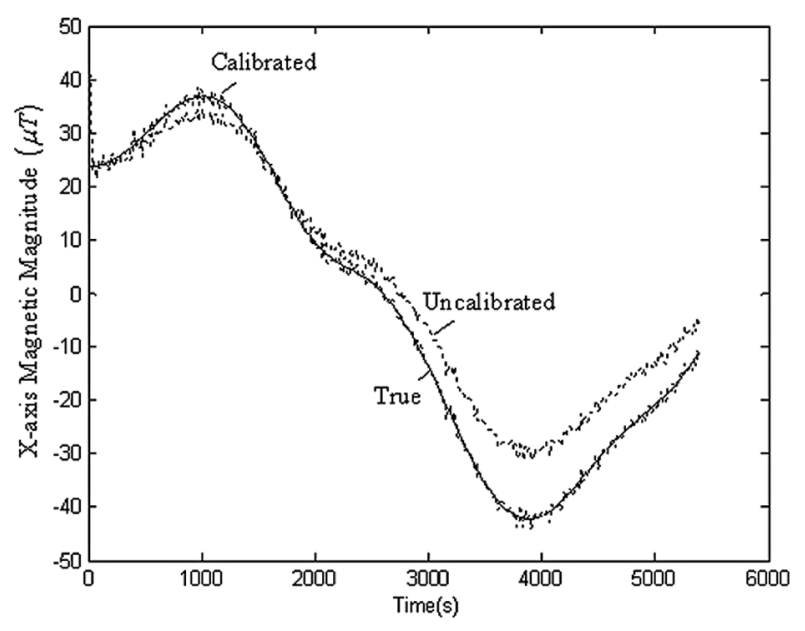

Fig. 8. $X$-axis geomagnetic field magnitude in body frame of the calibrated and uncalibrated magnetometers.

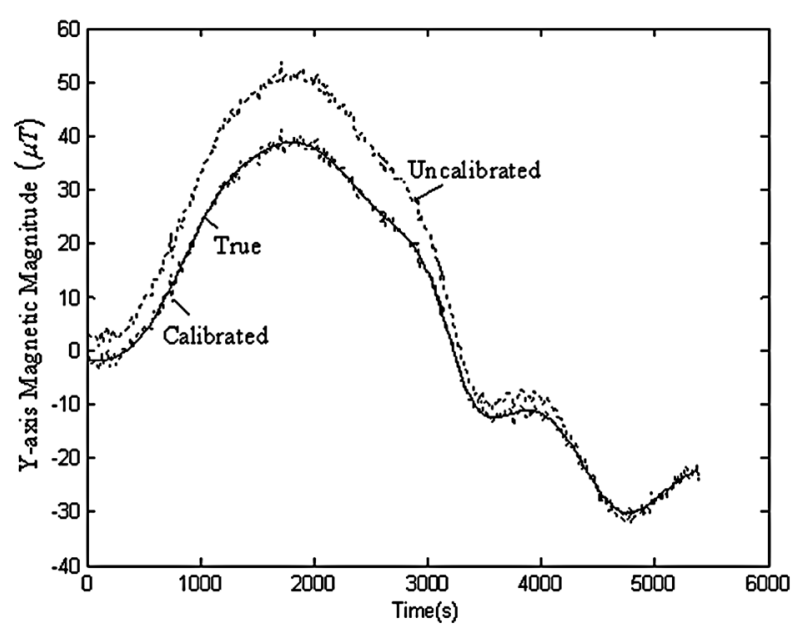

Fig. 9. $Y$-axis geomagnetic field magnitude in body frame of the calibrated and uncalibrated magnetometers.

\section{Conclusion}

Most of the pico and small satellites in low-earth orbit use magnetometers for the attitude determination. Magnetometers are lightweight, reliable and low power consumption sensors therefore they are very convenient as an

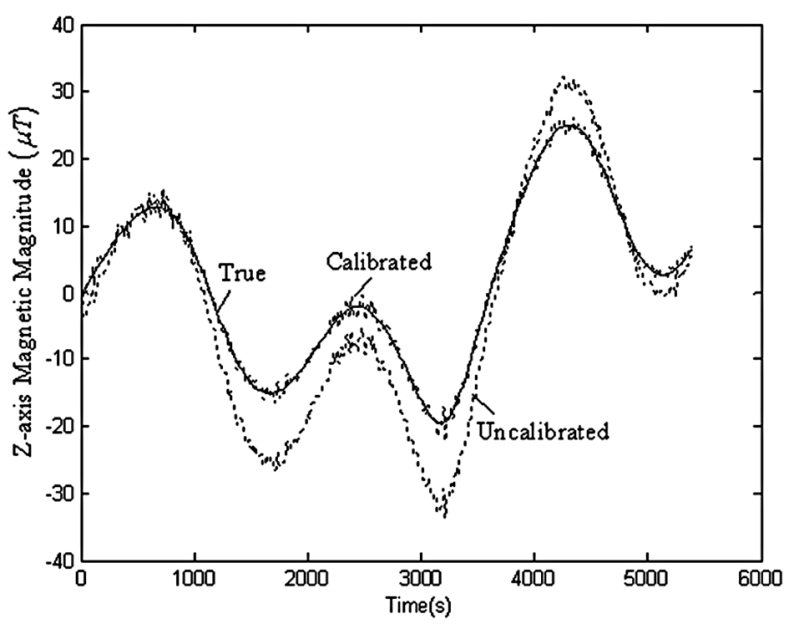

Fig. 10. $Z$-axis geomagnetic field magnitude in body frame of the calibrated and uncalibrated magnetometers.

attitude sensors for small satellites. In order to determine attitude accurately, scale factor and bias noise of magnetometer should be compensated. In initial phase of the satellite mission, measurement of magnetometers are indispensable for attitude determination and control system. In this study a Linear Kalman filter based algorithm for the estimation of magnetometer biases and scale factors is proposed.

For the operation of the proposed method, the attitude transformation matrix is needed to transform the orbit modeled geomagnetic vector to body coordinates. Measurements from other attitude sensors (for example, GPS, star sensors, etc.) are thus essential for the accuracy of this magnetometer bias and scale factor calibration algorithm.

Simulation results show that, it is possible to estimate both magnetometer bias and scale factor accurately via the presented LKF. Since magnetometer utilization has significance especially for Pico satellite missions, proposed algorithm may affect the mission performance and reliability in a considerable degree.

Acknowledgements. The work was supported by TUBITAK (The Scientific and Technological Research Council of Turkey), Grant 113E595. 
Table 2. Relative estimation errors of magnetometer biases and scale factors.

\begin{tabular}{ccccccc}
\hline Time $(\mathrm{s})$ & $\begin{array}{c}\text { Rel. Error } \\
R b_{x}(\%)\end{array}$ & $\begin{array}{c}\text { Rel. Error } \\
R b_{y}(\%)\end{array}$ & $\begin{array}{c}\text { Rel. Error } \\
R b_{z}(\%)\end{array}$ & $\begin{array}{c}\text { Rel. Error } \\
R S_{x}(\%)\end{array}$ & $\begin{array}{c}\text { Rel. Error } \\
R S_{y}(\%)\end{array}$ & $\begin{array}{c}\text { Rel. Error } \\
R S_{z}(\%)\end{array}$ \\
\hline 150 & 49.66 & 31.382 & 8.815 & 14.0375 & 76.5583 & 18.6 \\
750 & 28.605 & 1.562 & 0.4925 & 2.4375 & 0.8833 & 0.7642 \\
1500 & 15.37 & 1.688 & 0.0875 & 3.625 & 0.825 & 1.1785 \\
2250 & 0.2475 & 1.672 & 0.2975 & 3.3625 & 1.25 & 2.1142 \\
3000 & 0.095 & 1.828 & 0.6825 & 4.5125 & 0.8416 & 1.5857 \\
3750 & 0.2625 & 0.988 & 0.585 & 2.575 & 0.3833 & 1.5 \\
4500 & 0.295 & 1.226 & 1.4275 & 0.5875 & 0.9416 & 1.75 \\
5250 & 0.120 & 1.262 & 1.965 & 1.350 & 2.1166 & 1.2857 \\
5400 & 0.050 & 1.140 & 1.620 & 0.2875 & 1.2583 & 2.6357 \\
\hline
\end{tabular}

\section{References}

1. J.R. Wertz, Spacecraft attitude determination and control (Kluwer Academic Publishers, Dordrecht, Holland, 1988)

2. R. Alonso, M.D. Shuster, Two steps: A fast robust algorithm for attitude- independent magnetometer-bias determination, J. Astronautical Sci. 50, 433-451 (2002)

3. R. Alonso, M.D. Shuster, Attitude-independent magnetometer-bias determination: A survey, J. Astronautical Sci. 50, 453-475 (2002)

4. M.D. Shuster, R. Alonso, Magnetometer calibration for the first Argentine spacecraft, Adv. Astronautical Sci. 91, 29-46 (1996)

5. R. Alonso, M.D. Shuster, Centering and observability in attitude-independent magnetometer-bias determination, J. Astronautical Sci. 51, 133-141 (2003)

6. R. Alonso, M.D. Shuster, Complete linear attitudeindependent magnetometer calibration, J. Astronautical Sci. 50, 477-490 (2002)

7. E. Kim, H. Bang, Bias estimation of magnetometer using genetic algorithm, in Proc. Int. Conf. Control Automation and System 200\%, Seoul, Korea, 2007, pp. 195-198

8. J.L. Crassidis, K. Lai, R.R. Harman, Real-time attitudeindependent three-axis magnetometer calibration, J. Guidance Control Dynamics 28, 115-120 (2005)

9. L. Huang, W. Jing, Attitude-independent geomagnetic navigation using onboard complete three-axis magnetometer calibration, in Proc. 2008 IEEE Aerospace Conference, Montana, USA, 2008, pp. 1-7

10. W. H. Steyn, A Multi-mode Attitude Detarmination and Control System for Small Satellites, Ph.D. dissertation, University of Stellenbosch, South Africa, 1995
11. R. DaForno et al., Autonomous navigation of megSat 1: Attitude, sensor bias and scale factor estimation by EKF and magnetometer-only measurement, in Proc. 22nd AIAA International Communications Satellite Systems Conference and Exhibit, California, USA, 2004

12. G.F. Ma, X.Y. Jiang, Unscented Kalman filter for spacecraft attitude estimation and calibration using magnetometer measurements, in Proc. 4th International Conference on Machine Learning and Cybernetics, Guangzhou, China, 2005, pp. 506-511

13. C. Hajiyev, Orbital Calibration of microsatellite magnetometers using a linear Kalman filter, Measurement Techniques 58, 1037-1043. DOI : 10.1007/s11018-015-0838-4

14. P. Sekhavat, Q. Gong, I.M. Ross, NPSAT I parameter estimation using unscented Kalman filter, in Proceedings of 2007 American Control Conference (IEEE, New York, USA, 2007), pp. 4445-4451

15. T. Inamori, N. Sako, Sh. Nakasuka, Strategy of magnetometer calibration for nano-satellite missions and in-orbit performance. AIAA Guidance, Navigation, and Control Conference, 2-5 August 2010, Toronto, Ontario Canada, pp. $1-13$

16. R.E. Kalman, A new approach to linear filtering and prediciton problems, ASME J. Basic Eng. 35-45 (1960)

17. C. Hajiyev, F. Caliskan, Fault diagnosis and reconfiguration in flight control systems (Kluwer Academic Publishers, Boston, USA, 2003)

18. R.K. Mehra, J. Peschon, An innovations approach to fault detection and diagnosis in dynamic systems, Automatica 7, 637-640 (1971) 